\title{
A Novel Machine Learning Model Based on Exudate Localization to Detect Diabetic Macular Edema
}

\author{
Oscar Perdomo $^{1 *}$, Sebastian Otalora ${ }^{1}$, Francisco Rodríguez $^{2}$, John Arevalo $^{1}$, and \\ Fabio A. González ${ }^{1}$ \\ ojperdomoc@unal.edu.co,jsotaloram@unal.edu.co,fjrodriguez@fon.org.co, \\ jearevaloo@unal.edu.co,fagonzalezo@unal.edu.co \\ ${ }^{1}$ Mindlab Research Group, Universidad Nacional de Colombia, Bogotá, Colombia; ${ }^{2}$ National \\ Ophthalmological Foundation, Bogotá, Colombia
}

\begin{abstract}
Diabetic macular edema is one of the leading causes of legal blindness worldwide. Early, and accessible, detection of ophthalmological diseases is especially important in developing countries, where there are major limitations to access to specialized medical diagnosis and treatment. Deep learning models, such as deep convolutional neural networks have shown great success in different computer vision tasks. In medical images they have been also applied with great success. The present paper presents a novel strategy based on convolutional neural networks to combine exudates localization and eye fundus images for automatic classification of diabetic macular edema as a support for diabetic retinopathy diagnosis.
\end{abstract}

\section{Introduction}

Diabetes mellitus is one of the leading causes of death according to the World Health Organization (WHO) [1]. Diabetic retinopathy (DR) is a condition caused by prolonged diabetes mellitus, causing blindness worldwide in persons in their productive age (2069 years). One of the main problems associated to DR is that most people have no symptoms, and suffer the disease without being diagnosed [1,2]. DR is one of the microvascular complications of diabetes, which affects the small vessels of the retina (the innermost eye light sensitive layer), being a major cause of blindness. Diabetes macular edema (DME) is a complication of DR that occurs when the vessels of the central part of the retina (macula) are affected by accumulation of fluid and exudate formation in different parts of the eye[3]. DME classification is performed as follows:

- Class 0: No signs of retinopathy.

- Class 1: Mild. Presence of exudates in the macula, but without affecting the central macular area.

- Class 2: Moderate. Presence of exudates in central macular region without affecting the fovea.

- Class 3: Severe, Retinal thickening or presence of exudates that affect the foveal area $[4,5]$.

X. Chen, M. K. Garvin, J. Liu, E. Trucco, Y. Xu (Eds.): OMIA 2016, Held in Conjunction with MICCAI 2016, Athens, Greece, Iowa Research Online, pp. 137-144, 2016. Available from: http://ir.uiowa.edu/omia/2016_Proceedings/2016/ 
Eye fundus image is a common clinical exams that presents some advantages as: high patient compliance, dilation not required in most cases, quick and simple technique, images can be saved and analyzed at a later time, and progression of diseases can be monitored over time[6,7,8,9]. A faster diagnosis of the disease grade may help to a proper clinical treatment, improving the patients quality of life. Macular exudates can be detected by examining color photographs of the back of the eye, which is interpreted by a specialist ophthalmologist in retina. This process has some drawbacks such as being very time consuming and repetitive for clinical personnel, depending of the ophthalmologist's experience, and its susceptibleness to inter-observer variability. Moreover, the analysis of lots of images without any pathologies increases the work time, but decreases the time of analysis to others images with pathologies. Computer-aided systems (CADx) are an interesting alternative to tackel these problems CADx systems perform an automatic assesment of the disease grade, they may increase the number of patients diagnosed and may reduce the time to detect diabetic eye diseases [10].

Some studies have been done with different algorithms for automatic DME assessment. Giancardo et al[11], used a feature vector representing the exudate probability map along with a wavelet decomposition and classifier for for automatic lesion segmentation and DME assessment. Vasanthi and Banu [12]studied the preprocessing of images and the use of an adaptive neuro-fuzzy inference system and an extreme learning machine to classify eye fundus images between normal and with some kind of DME. Ramya and Vijayprasath[13] proposed the use of a Gaussian data descriptor and principal component analysis data to classify normal and abnormal retinal images. Kunwar et al [14], presented a method for texture extraction from regions of interest of the eye fundus image and combined it with a support vector machine classifier. This paper presents a novel deep learning method for DME grade assessment based on a two stage convolutional neural network that detects regions with exudates in the input image and predicts its DME class. The method was evaluated in a publicly available dataset and shows competitive results when compared to state-of-the-art methods.

The remainder of this paper is organized as follows: First, in Section 2, an overview of our convolutional neural network based method is presented. Then in section 3 the datasets and the evaluation of our method are described. In section 4, the experimental results are presented and discussed. Finally, section 5 draws conclusions and future work.

\section{Methods}

An overall view of our method is shown as a block diagram in Figure 1. Our method is based on convolutional neural networks. For successful training of deep neural networks a large number of training samples is required[17], in our case we need to have enough healthy and exudate samples, nevertheless, as is usual in many medical tasks, here the number of samples is limited and the class imbalance between the healthy and exudate patches is important and needs to be addressed. A standard way to obtain more samples is to rotate and flip the image, this was the data augmentation used in our experiments and is described in more detail in Section 2.1 
Our method (ED-DME classification) works in two stages. In the first one, the training of an exudate-detection model is done. This model is composed of eight layers using the e-ophtha database with RGB patches of $48 \times 48$ pixels as input. The second stage is the processing of the messidor database with 1200 images of size $512 \times 512$ pixels to generate a new dataset of 1200 grayscale mask images using the previous trained CNN model as predictor. The final stage is to train a DME-detection model based on the AlexNet architecture [17], which is composed of 17 layers using the RGB eye fundus images of $512 \times 512$ pixels plus the previous generated grayscale masks as the 4 -th input channel. One interesting aspect of our model is that incorporates the mask of exudates detection trained in the first stage to serve as input to the DME-detection model, thus, giving more insights to the later model of what kind of patterns are we looking for.

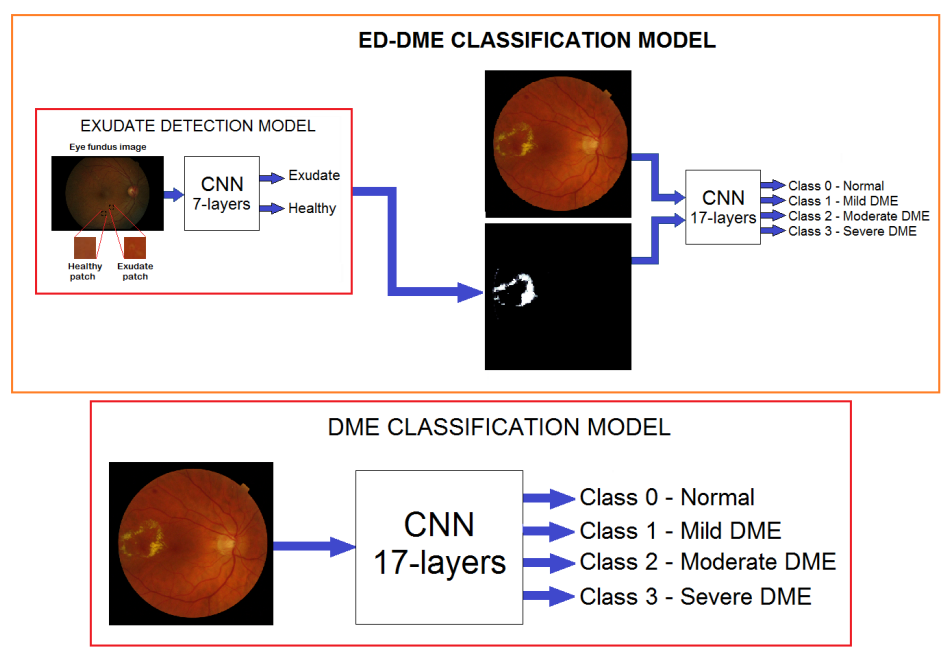

Figure 1. Block diagram with the proposed ED-DME Classification model and the DME classification model.

\subsection{Preprocessing}

In the preprocessing stage the application of a set of transformations that helps to improve performance in the following stages is made. The first step is to envelope the exudate in a bouding box in order to extract the Region of interest (ROI) from the eye fundus image of e-ophtha database in order to train the exudate-detection model. Secondly, we define an oversampling strategy using to get more patches and help to prevent overfitting during training, this oversampling step showed significant improvements in our experimentation in comparisson with no doing it. Finally, the resize of all the Messidor database images to a $512 \times 512$ sized images, keeping the aspect ratio. 
Cropping The exudates labels in e-ophtha dataset were manually segmented by three expert opinions for each examination on this database. According to the mean of the lesion's size of 2136 exudates, the patch's size was fixed of $48 \times 48$ pixels. An exudate patch presented a size greater than the $60 \%$ of the ROI. Otherwise, the patch was labelled as healthy patch.

Data augmentation Data augmentation was the strategy used to decrease the possibility of overfitting during the training of the networks creating artificial samples[17]. The combination of two flipping and rotation of 90, 180 and 270 degrees was made in order to generate 7 new label-preserving samples to the e-ophtha dataset. On the other hand, flipping left to right and up to down plus the rotation of 90, 180 and 270 degrees in order to generated 5 new label-preserving samples for the classes 1 (mild DME) and 2 (moderate DME) and the combination of two flipping to rotated images of 45,90 , $135,180,225,270$ and 315 degrees in order to generated 15 new label-preserving samples for the class 3 (severe DME) to the Messidor dataset. The main advantage of data augmentation is the ability of the model to learn from such transformations.

\subsection{Exudate-detection model}

Convolutional Neural Networks (CNN) a type of bio-inspired artificial neural network based on the animal visual cortex. CNN are trained with the ability to respond to the variability and invariant to the exact position of the pattern in order to detect visual patterns with minimal preprocessing. The architecture of the CNN contains convolutional, pooling, and fully connected layers. Convolutional layer is a set of learnable filters that convolved computing the dot product between the filter and the input generating an activation map of that filter using a stride with a kernel size that represent the receptive field. Pooling layer is a non-linear function used to reduce the number of variables of the convolutional layer but extracting. Max Pooling is the maximum value in a windows define by a kernel size with a stride. Fully-connected layer connects the whole number of neurons among them at previous layer[16].

\subsection{DME classification model}

The architecture of this network is summarized as follows. It contains eight learned layers, six convolutional and three fully-connected. Below, we describe convolutional, pooling and fully-connected layers, but this architecture has normalization layers proposed according bioinspired-based on inhibition schemes presented in the brain. The activations of normalization layer applies a transformation that maintains the values of mean activation close to 0 keeping the activation standard deviation close to 1 . [17]

\section{Experimental setup}

\section{1 e-Ophtha Dataset}

The e-ophtha database contains 47 color fundus images with size ranging from $1440 \times$ 960 to $2540 \times 1690$ pixels, which were segmented in order to find exudates by three 
ophthalmologists from the OPHDIAT Tele-medical network under the the French Research Agency (ANR) project, specially designed for scientific research in Diabetic Retinopathy (DR) [15]. The labelled patches dataset created of $48 \times 48$ pixels with exudate and healthy classes after the preprocessing steps: cropping and data augmentation was randomly split by images where an image could only belong to a group with the following datasets distribution: Training dataset with 8760 patches by each class, validation dataset has 328 by class and test dataset has 986 by class, representing $45 \%$, $15 \%$ and $40 \%$ respectively.

\subsection{Messidor Dataset}

The Methods to evaluate segmentation and indexing techniques in the field of retinal ophthalmology or Messidor database contains images of the posterior pole of eyes of subjects diagnosed with Diabetes Mellitus, no data with respect to time of disease progression, sex or age of the subjects. Messidor is a research program funded by the Ministry of Research and Defense of France in a program TECHNO-VISION 2004. The database contains 1200 Messidor color photos Fundus provides information on the posterior pole. It was acquired by three ophthalmologic departments using a 3CCD color video camera in a retinograph TRC NW6 Topcon non-mydriatic a $45^{\circ}$ field of vision. The images were captured using 8 bits per color plane images with ranges of $1440 \times 960,2240 \times 1488$ and $2304 \times 1536$ pixels. 800 images were acquired with pupil dilation (with a previous drop of tropicamide $0.5 \%$ ) and 400 without dilation. The International Clinical Disease Severity Scale for DR with regard to diabetic macular edema was performed by ophthalmologist from National Ophthalmological Foundation to classifiy as mild, moderate and severe according on the distance of the exudates and thickening from the center of the fovea and normal without any exudates. The results after the classification to the four classes[4,5] presented 878, 140, 146 and 36 images respectly. After data augmentation stage for classes 1 and 2 and 3 applied to training dataset, the three sets with the following distribution: Training dataset with $2215 \mathrm{im}$ ages, validation dataset has 121 by class and test dataset has 239 by class, representing $70 \%, 10 \%$ and $20 \%$ respectively.

\subsection{Evaluation}

The three classification models (ED, DME and ED-DME) were trained using stochastic gradient descent. The learning rate and batch size parameters were explored in a grid search manner and their values are listed in Table 1, using 30 as the number of epochs to train the model. The results of DME classification CNN was chosen as baseline. In this $\mathrm{CNN}$, the performance was $92 \%$ in sensitivity and $40.6 \%$ in specificity applied in a image-based experimental setup using only the Messidor dataset. The proposed approach was implemented with Python 2.7 and the interactive NVIDIA Deep Learning GPU Training System (DIGITS) using GeForce GTX TITAN X from NVIDIA. During all the experiments, training loss and validation loss, as well as the accuracy over the validation set were monitored. 


\section{Results}

Results of the systematic exploration are reported in Table 1. The best performance of the ED model was obtained with a learning rate of 0.01 and a batch size of 64 , and the best performance of DME-classification model was obtained with a learning rate of 0.01 and a batch size of 32 .

Table 1. Performance of ED and DME models with different batch size learning rates in training dataset

\begin{tabular}{|c|c|c|c|c|c|}
\hline CNN & Learning rate & Batch size (train) & Loss(train) & Loss(val) & Accuracy(val) \\
\hline \hline ED model & $\mathbf{0 . 0 1}$ & $\mathbf{6 4}$ & $\mathbf{0 . 0 0 3 4}$ & $\mathbf{0 . 0 0 0 2}$ & $\mathbf{9 9 . 8 0}$ \\
\hline ED model & 0.001 & 64 & 0.4550 & 0.4763 & 80.00 \\
\hline ED model & 0.00001 & 64 & 0.5850 & 0.5956 & 70.39 \\
\hline DME model & $\mathbf{0 . 0 1}$ & $\mathbf{3 2}$ & $\mathbf{0 . 8 5}$ & $\mathbf{0 . 7 8}$ & $\mathbf{7 7}$ \\
\hline DME model & 0.001 & 32 & 0.915 & 0.825 & 73.5 \\
\hline DME model & 0.0001 & 32 & 1.388 & 1.301 & 64.5 \\
\hline
\end{tabular}

We assessed the ability of the ED model with the best parameters to create a mask from the test dataset that was randomly selected. Figure 2 presented an eye fundus image of $1440 \times 960$ pixels, ground truth generated by expert ophthalmologist and the mask generated using the exudate-detection CNN to classify patches as exudates if the output of the softmax layer is above 0.75 ; otherwise, it was classified as a healthy patch.
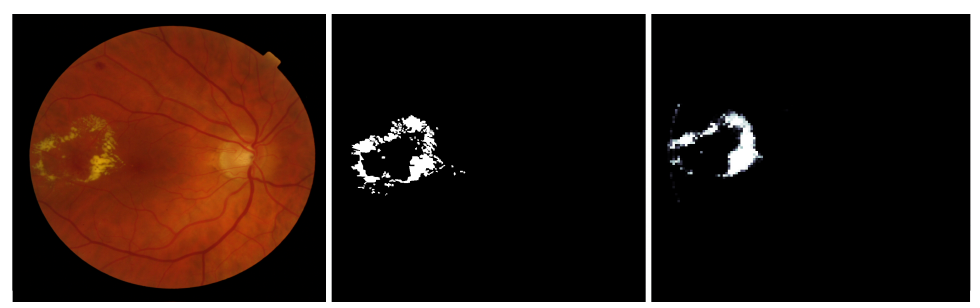

Figure 2. Eye fundus image with DME from test dataset, ground truth generated by expert ophthalmologist and exudates automatic label by exudate-detection model

Then, we validated the proposed method comparing the results of the DME and ED-DME classification models applied to the test dataset. In Table 2 is reported the confusion matrix of the two methods.

Finally, in Table 3 is reported the performance measures of sensitivity and specificity of the two methods. The proposed method clearly outperforms in detection of classes 0,1 and 2 . This shows that the proposed method is able to better capture the visual features that characterize exudates to classify diabetic macular edema. 
Table 2. Confusion matrix of the proposed method (ED-DME) compared to the DME model.

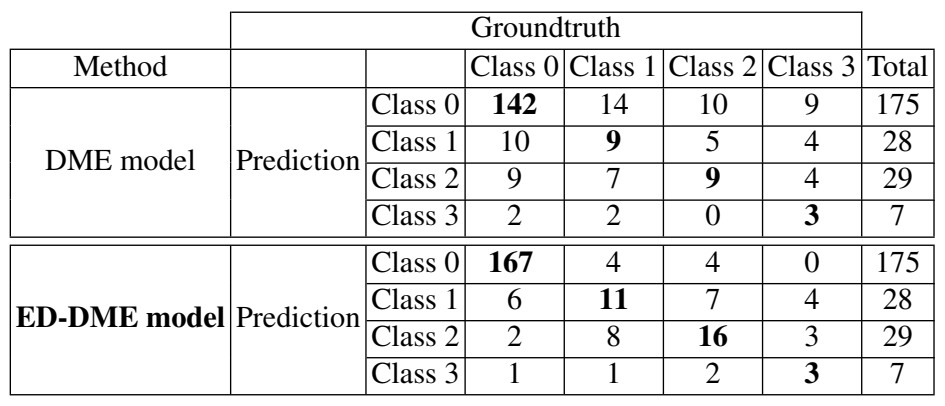

Table 3. Performance measures per class for the proposed method (ED-DME) compared to the DME-model.

\begin{tabular}{|c|c|c|c|}
\hline Method & & Sensitivity (\%) & Specificity (\%) \\
\hline \multirow{4}{*}{ DME model } & Class 0 & 87.1 & 56.6 \\
\cline { 2 - 4 } & Class 1 & 28.1 & 90.8 \\
\cline { 2 - 4 } & Class 2 & 37.5 & 90.6 \\
\cline { 2 - 4 } & Class 3 & 15.0 & 98.1 \\
\cline { 2 - 4 } & MEAN +/- STD & $41.9+/-31.5$ & $84.0+/-18.6$ \\
\hline \multirow{4}{*}{ ED-DME model } & Class 0 & 94.9 & 87.3 \\
\cline { 2 - 4 } & Class 1 & 45.8 & 92.0 \\
\cline { 2 - 4 } & Class 2 & 55.2 & 93.8 \\
\cline { 2 - 4 } & Class 3 & 30.0 & 98.2 \\
\cline { 2 - 4 } & MEAN +/- STD & $\mathbf{5 6 . 5 + / - 2 7 . 6}$ & $\mathbf{9 2 . 8 + / - 4 . 5}$ \\
\hline
\end{tabular}

\section{Discussion and conclusion}

We have presented a novel eye fundus classification model composed of two CNNs which are able to detect effectively the patterns in the 4 classes of diabetic macular edema.

The experimental results showed that the proposed method is highly effective to detect normal subjects in eye fundus images. The results clearly improved the sensitivity and specificity results produced by the single DME-method, thanks to the ability of the proposed method to first locate exudates, generating information as a mask in the input layer to improve the classification of DME. The proposed method is a good option to detect other diseases where the previous detection of abnormalities may improve the disease classification. Other kind of images of the eye, such as, angiography mask with the vessels segmentation may improve the performance to classify other ophthalmological disease, such as diabetic retinopathy.

Early detection of clinical signs in macular edema may improve diagnosis of the disease and the classification between the different stages of DME. This research is a first step in building telemedicine tools that can support medical personnel for detecting ophthalmic diseases using convolutional neural networks in eye fundus images. 


\section{Acknowlegement}

Oscar Perdomo thanks to COLCIENCIAS for funding this research in a doctoral grant. The authors also thank to the National Ophthalmological Foundation for supporting the project

\section{References}

1. World Health Organization (WHO), Diabetes Programme. 2016, Available in: http://www.who.int/diabetes/en/, 5th edition, 2016.

2. International Diabetes Federation (IDF), IDF Diabetes Atlas Update 2015, 7th edition. Available in: http://www.idf.org/diabetesatlas.

3. R. Bernardes, Cunha-Vaz, José. Optical Coherence Tomography: A clinical and technical update. n.32, vol. 2, pp. 1-21, 2012.

4. $\mathrm{L}$. Wu, et al. Classification of diabetic retinopathy and diabetic macular edema. World $\mathrm{J}$ Diabetes. vol. 4, n. 6, pp. 290-294, 2013.

5. J. Ding and T. Y. Wong. Current epidemiology of diabetic retinopathy and diabetic macular edema. Curr. Diabetes Rep., vol. 12, no. 4, pp. 346-54, 2012.

6. Yau JW, et al, Global prevalence and major risk factors of diabetic retinopathy, Diabetes Care, vol. 35, n. 3. pp. 556-64, 2012.

7. Stitt AW, et al. Advances in our understanding of diabetic retinopathy, Clin Sci (Lond), vol. 125, n. 1. pp. 1-17, 2013.

8. Kenneth W., et al. Detection of Anatomic Structures in Human Retinal Imagery, IEEE Transactions on medical imaging, vol. 26, n. 12. pp. 1729-1739, 2007.

9. A.P. Shingade, A.R. Kasetwar. A review on implementation of algorithms for detection of diabetic retinopathy, IJRET: International Journal of Research in Engineering and Technology, vol 3, n. 3, pp. 87-94, 2014.

10. van Grinsven M, van Ginneken B, Hoyng C, Theelen T, Sanchez C. Fast convolutional neural network training using selective data sampling: Application to hemorrhage detection in color fundus images. IEEE Trans Med Imaging, 2016.

11. L. Giancardo, et al. Exudate-based diabetic macular edema detection in fundus images using publicly available datasets. Med Image Anal, vol. 16, n. 1, pp. 216-26, 2012.

12. S. Vasanthi and R. S. D. W. Banu. Automatic Segmentation and Classification of Hard Exudates to Detect Macular Edema in Fundus Images. J. Theor. Appl. Inf. Technol., vol. 66, no. 3, pp. 684-690, 2014.

13. M. Ramya and S. Vijayprasath. An Effective Analysis of Macular edema Severity for Diabetic Retinopathy. IJIRSET, vol. 3, no. 3, pp. 739- 746, 2014.

14. A. Kunwar,S. Magotra, M. P. Sarathi. Detection of High-Risk Macular Edema using Texture features and Classification using SVM Classifier. Advance in computing communications and informatics (ICACCI). pp. 2285-2289, 2015.

15. Decencieriere et al, TeleOphta: Machine learning and image processing methods for teleophthalmology, IRBM -Special issue:ANR TECSAN: Technologies for Health and Autonomy, vol. 34,n. 2, pp. 196-203, 2013.

16. LeCun Y. et al, Gradient-based learning applied to document recognition, Proc of the IEEE, vol 86, n. 11, pp.2278-2324, 1998.

17. Krizhevsky, A., I. Sutskever and G. Hinton, ImageNet Classification with Deep Convolutional Neural Networks, Proc. Neural Information and Processing Systems, 2012. 